PROCEEDINGS OF THE SIXTH INTERNATIONAL SEMINAR ON

"Recent Research and Design Progress in Aeronautical

Engineering and its Influence on Education " - Riga, Latvia

\title{
DYNAMICS OF AN ELASTIC AIRPLANE
}

\section{P. Chudý, V. Daněk}

Institute of Aerospace Engineering, Brno University of Technology, Technická 2, Czech Republic.

E-mail: peter.chudy@pobox.sk; danek@fme.vutbr.cz

Received 2211 2004, accepted 17032005

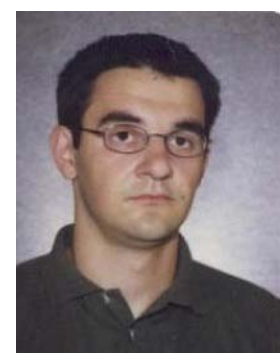

Peter CHUDÝ, PhD student

Education: 2001 -Brno University of Technology, Institute of Aerospace Engineering.

Affiliations and functions: Since 2001 - Ph.D. student - Institute of Aerospace Engineering, Brno University of Technology.

Experience: 2001-2002 CFD analysis of Turboprop Engine Nacelle; 2002-2004 computational flight dynamics, estimation of aeroelastic response.

Present position: $\mathrm{PhD}$ student at the Institute of Aerospace Engineering, Faculty of Mechanical Engineering; Brno University of Technology; Technická 2, 61669 Brno, Czech Republic;

Tel.: +420 54114 3370, E-Mail.: peter.chudy@pobox.sk

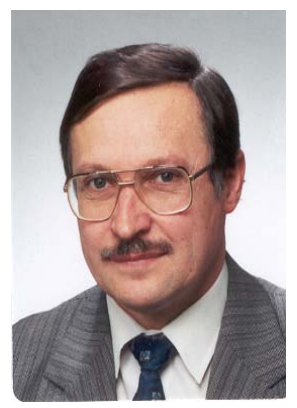

Vladimír DANĚK, Assoc Prof

Education: 1970 - Faculty of Aviation, Military Academy of Brno, M.Sc.(Eng.) 1970, Ph.D. 1981, Assoc Prof. 1986. Affiliations and functions: LET Kunovice - Aerodynamicist (1970-1974); Military Academy of Brno, Faculty of Aviation Engineering - Lecturer of Aircraft Design (1974-1983); Brno University of Technology, Faculty of Mechanical Engineering (FME), Institute of Aerospace Engineering (IAE) - Senior Lecturer (from 1983), Vice-Dean of FME (1991-1994).

Professional memberships: Member of the Czech Society for Mechanics at the Academy of Science of the Czech Republic; Member of Technical Committee of Czech Aeroclub.

Experience: Aerodynamics and flight mechanics research and development worker since 1970; teaching aircraft design, aerodynamics, flight mechanics, aero elasticity, flight-testing; Test pilot qualification for glider and motor glider.

Present position: Senior Lecturer of Flight Mechanics, Vice-Director of IAE, Head of Flight Research and Testing Group of IAE, Faculty of Mechanical Engineering, Brno University of Technology, Technická 2, 61669 Brno, Czech Republic; Tel.: +420 54114 2229, E-Mail: danek@fme.vutbr.cz

Abstract. This paper presents the work performed by the Institute of Aerospace Engineering at the Brno University of Technology. The purpose of the project was to compare the results obtained from classical analytical solutions and a complex numerical simulation of an airplane's aero elastic response. Compared to the analytical solution, which reduces the entire process to a straightforward manipulation with time-proven graphs and tables, the numerical simulation offers a more complex description of the dynamic processes. A complex simulation, in contrast to the analytical solution providing us with only one estimated parameter, allows monitoring selected quantities in the time domain, thus giving us a tool for a visual qualification of the investigated process. In the past, dynamic aeroelastic properties were estimated utilizing simplified stick beam models. The desire for more complex aero elastic simulations led to the concept of the advanced aero elastic model, coupling advanced 3D structural FEM models with proven aerodynamic theory in the form of the DLM panel method.

Keywords: computational aero elasticity, gust loading, dynamic response estimation, finite element model, unsteady aerodynamics, advanced modeling techniques.

\section{Nomenclature}

$\left[M_{h h}\right]$

$\left[K_{h h}\right]$

$M$

$K$

c

$\left[Q_{h h}(m, k)\right]$

$\omega$

$f$

$g$

$\rho$
$V$
$\left\{u_{h}\right\}$
$\{P(\omega)\}$
$U_{0}$
$a$
$m$
$S$
$k$
$\mu$
$l_{m}$

Air density

Modal Mass Matrix

Modal Stiffness Matrix

Mach Number

Reduced Frequency

Reference Length

Unsteady Aero Force Matrix

Circular Frequency

Frequency

Structural Damping
Airplane Velocity

Modal Amplitude Vector

Applied Gust Loading

Gust velocity

Slope of wing lift curve

Mass of the airplane

Design wing area

Gust alleviation factor

Non-dimensional mass ratio

Mean geometric chord 


\section{Introduction}

Estimation of the dynamic response qualities is one of the crucial tasks in aerospace engineering. Prior to the period of numerical simulation, all the estimations were done using data derived from flight measurements of airplanes that were already built. Increase in computational power and advances in numerical mathematics pushed the estimation procedures far beyond what many believed would be possible to achieve. Modern concepts utilize the advantages of already proven computational methods and combine them to create a far better virtual representation of the investigated system. The necessity to extend the dynamic analysis beyond the classical approaches arose from the changing requirements on aeronautical structural design motivated mainly by advances in aerodynamics and material sciences. As the main driving force of airplane designers hides behind the word efficiency this became the driving factor for slender wing design, which in turn imposed new problems in flight dynamics. Slender wings are more likely to be subjected to larger deformations, thus leading to potential load redistributions and variations in dynamics resulting in changes in handling qualities.

\section{Airborne System Modeling}

Suitable aero elastic models consist of two major parts: the first represents aerodynamic forces acting on the structure (referred to as the aerodynamic model) and the second represents dynamic characteristics of the structure (usually carries the designation 'dynamic' or elastic-inertial model) [1, 2]. However, making it work requires the utilization of techniques that transform the motion of the grid points of the aerodynamical part to deflections of grid points used by the dynamic representative. It sounds logical when speaking of aero elastic models that the subject is aero elasticity oriented. However, the intention of our extended research is to pursue the area of classical aeroelastic computation and put it further towards modeling the flying qualities of aircrafts, stability and control - in other words mathematical modeling of elastic aircraft's flight dynamics.

\section{Unsteady Aerodynamics}

It is understood that to create a reliable aeroelastic model, a huge amount of topical related data needs to be acquired. Also the tasks of creating the sub-models (aero or elastic-inertial) have their own finesses. The straightforward the structural model's physical boundaries could be modeled, the more complicated is the "description" of the surrounding flow field. For a long time, aerodynamic characteristics have been evaluated using various panel methods, which, according to the level of acceptable simplification, neglected some basic geometrical characteristics of lifting surfaces and bodies imposed to the airflow. From certain points the panel methods seem to be outdated and not worthy of further implementation. However, compared to the more advanced and more sophisticated finite volume algorithms, the panel methods offer reasonable results without the time, storage and computational penalty. Utilizing specific mathematical concepts to evaluate aerodynamic loading acting on the surface suggests revision of the nature of the flight conditions. Panel methods based on the potential flow theory are not able to capture flow separation effects frequently occurring during maneuvers at lifting surfaces. To capture the effects of flow non-linearity's corrective methods need to be implemented. Utilization of panel method based aerodynamic modeling techniques heavily relies on their time efficiency and overall simplicity. Even though advanced coupling methods to join complex structural and aerodynamic model have been developed, implementation of a bit less sophisticated couple was found suitable for the initial research attempt.

\section{Structural representation}

Use of detailed structural models was previously limited to cases of static or dynamic load tests. These have been compared to experimentally obtained data and calibrated. A generally accepted illustration of classical aeronautical structure was projected to the simplified beam model. Long slender wings, tubular fuselages, monocoque or semi-monocoque constructions allowed using the beams instead of complex full 3D bodies. These simplifications proved to be adequate for some groups of computational tasks. Creating a beam model requires a qualified guess on which of structural elements should be inherited in the simplification. Even if this might sound promising, the method seems feasible and acceptable only for slender aeronautical structures, thus eliminating the possibility to use this modeling technique for delta wing platforms or flying wing concepts. The idea behind using a more complex structural representation finds its roots in the search for one advanced model that could be used for several sets of tests, ranging from simple static loadings to dynamic maneuver response evaluations. However, the more complex the description of the model gets, the more obstacles can be expected in the computational process. Besides their overall simplicity, the 'beam' models offer a great advantage in straightforward modal calibration. The complex geometry of an airplane modeled in terms of finite elements tends to create significant concerns in the modal analysis part of the computational process where parasitic vibration modes may accompany the process. Even the simple 'beam' representative can be treated by some simpler techniques; its complex 'relative' is basically sentenced to the very own idea of complex modeling. This technique covers all significant details that can be found in the structure, thus limiting the area of possible misjudgments. Considerations of all major structural elements surrounded by additional structural details should offer a relatively solid basis for modal based computational approaches.

\section{Modeling techniques}

To avoid the occurrence of noise in the form of parasitic vibration modes, the main modeling process focuses on the primary structure 'overseeing' the non- 
stressed parts. The term overseeing is limited to the extent of neglecting their load carrying capability and substituting geometrical and inertial properties into simpler forms. Typically, this technique replaces some airframe features such as engine covers, wheel doors, and large isolated masses such as the undercarriage, engine, onboard equipment, or non-stressed larger parts of fuselage. This approach is visually illustrated in the following series of pictures.

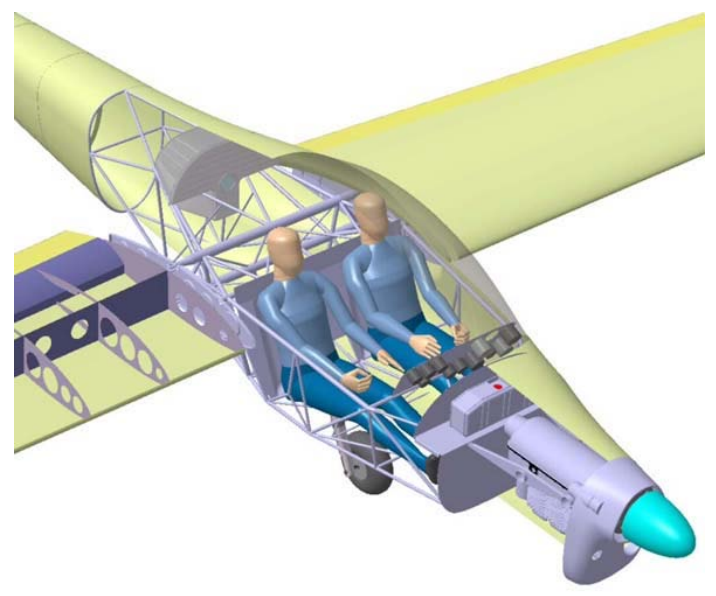

Fig 1. CAD model

To simplify the model of main fuel tank a simplified representative has been designed. The actual geometry of the main tank was inserted into a CAD program.
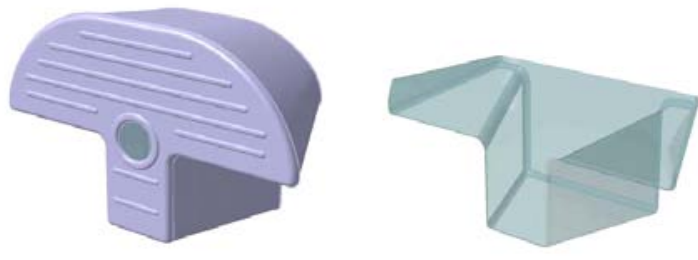

Fig 2. CAD model of fuel tank

Further steps involved 'filling' the tank with what was intended to be the 'liquid' fuel part. Adding appropriate physical characteristics offered the system an output in the form of relevant inertial characteristics and related mass properties. However this concept seems to be lengthy and time-consuming regarding the need to deliver all required data for your computations by your own means.

Considering the fact that the majority of future designs are born in the virtual world of CAD, this shouldn't impose any problem for future dynamic calculations. What seems to be a time penalty during the modeling phase pays back during the computational process. The main purpose is to create as realistic a dynamic model as possible while considering all the restrictions in computational capacity and the time dimension of the problem. Using simplified representatives of parts that are of secondary interest accelerates the computational phase.

\section{Technical and Design Data}

The airplane is a side-by-side two seat motorized mid wing glider with metallic structure. The fuselage is a semi-macaque stiffened with longerons. The wing is equipped with ailerons and landing flaps. It features, according to its classical design look, a main spar and auxiliary spar as load carrying elements. The stressed skin is metallic and riveted to the system of ribs and spars. Sufficient structural stiffness was achieved by adding a system of stringers. The tail unit was designed following the same principles. The fuselage features a fully equipped two-seat cockpit.

\section{FEM Modeling}

Figure 3 shows the resulting MSC/NASTRAN full span model that was used in the analysis. The complex model integrates all major structural features of the airframe. The model mass is continuously distributed over the entire structure. In special cases involving masses of non-load carrying elements, these masses are represented by concentrated masses respecting their actual physical position on the airplane. Control surfaces and high lift devices have been modeled as separate features using the same principles vital for the primary structure. This can be seen in Figure 3. Spines have been used to transfer modal displacements onto aerodynamic surfaces. The structural mesh of the wing includes elements representing the main and auxiliary spars, ribs, skin panels, and individual groups featuring ailerons and landing flaps. The structural design of control and high lift devices has a common classical core. The actual control surfaces and landing flaps are covered with nonstressed impregnated fabric and their load carrying capability was thus neglected.

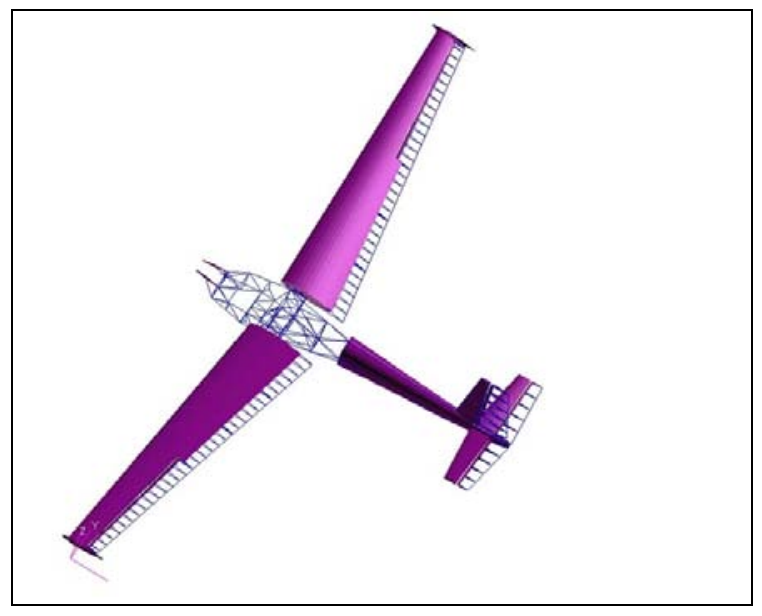

Fig 3. FEM model

\section{Aerodynamic panel method}

Doublet-Lattice Method (DLM) defines the aerodynamic conditions. The wing was divided into dorsal and ventral parts. The horizontal and vertical tails also feature aerodynamic panels. Separate aerodynamical panes have been added to the control surfaces and high lift devices. 
The modal displacements of aerodynamic boxes are related to the displacements of the structural grids by a surface splinting technique. The aerodynamic theory (DLM) used in this case does not allow the definition of camber, twist, or angle of incidence.

The theoretical basis of the DLM used to compute the unsteady aerodynamics is linearized aerodynamic potential theory. All lifting surfaces are assumed to lie parallel to the flow.

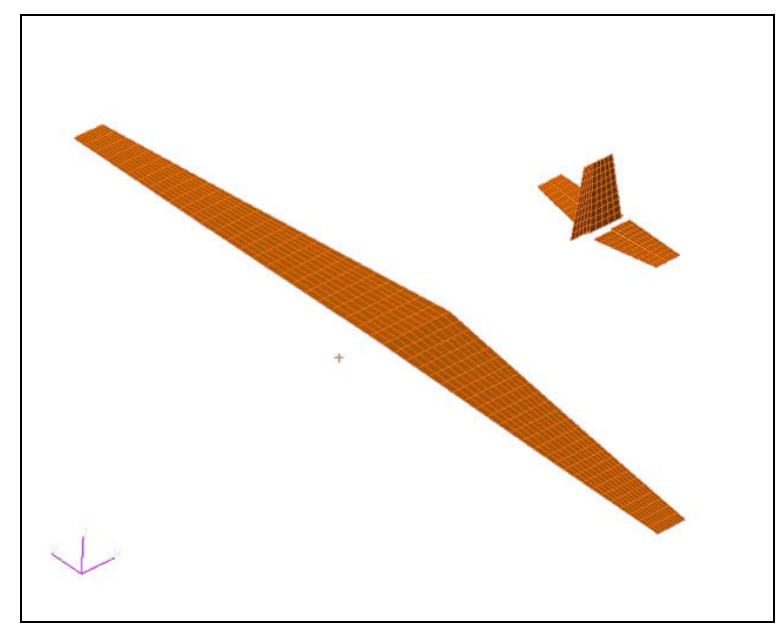

Fig 4. Aerodynamic panels

\section{Basic gust studies}

In general, there are two methods widely accepted by $\mathrm{e}$ aeronautical authorities. Both of them are implemented in the leading sets of international aeronautical safety requirements. The deterministic method describes the 'worst case' atmospheric gust approach. The nature of the gust field is reduced to a form of one dominant discrete gust with closer shape specification. The second approach belongs to the family of statistical methods. The atmosphere is described by the power spectral density function and transforms the problem from the time to a frequency domain. For both methods a set of gust velocities for specific flight conditions has been defined [9, 104$]$.

\section{Discrete gust analysis}

Considerable evidence can be found indicating that most of the severe gusts occur more or less as individual gusts. The deterministic computational method describes the 'worst case' atmospheric gust approach, where the nature of the gust field has been reduced to a form of one dominant discrete gust with closer shape specification. As stated at the beginning of this paper, the gust loads, due to a discrete gust, have usually been evaluated using static Pratt-Walker formula [9]. This formula became, for a long time, the basic tool for gust load evaluation. In the calculation of the static gust loads, a single gradient distance of 12.5 chords is specified. The actual gradient distance is not very important from the static gust viewpoint. The JAR 22 gust load formula for vertical gust is essentially:

$$
n=1 \pm\left[\frac{\frac{k}{2} \cdot \rho_{0} \cdot U_{0} \cdot V \cdot a}{\frac{m \cdot g}{S}}\right]
$$

in conjunction with:

$$
k=\frac{0.88 \cdot \mu_{g}}{5.3+\mu_{g}} \quad \mu_{g}=\frac{2 \cdot \frac{m}{S}}{\rho \cdot l_{m} \cdot a}
$$

Inclusion of the inertia forces associated with elastic-mode accelerations indicates dynamic gust loads. Since it is not clearly defined how the gust velocity varies with the gradient distance, the dynamic load determination for the glider gust response was performed using only a 12.5 chord gust profile. The excitation waveform was used in the following form:

$$
U=\frac{U_{0}}{2}\left[1-\cos \left(\frac{\pi \cdot V}{H \cdot t}\right)\right]
$$

Lifted or plunged vertically during uniform discrete gust, the glider encounters little inertia resistance. Conversely, accelerations in response to discrete gusts tend to be larger than they are for heavier transports. The essential feature of the analysis is the time history solution of the response.

\section{Analysis method}

Unsteady modal aerodynamic forces and gust loads are calculated in the frequency domain. Structural stiffness, mass, and damping are modeled using rigid body and elastic modes with structural damping. The resulting aeroelastic modal equations of motion can be written in the form [3]:

$$
\left[-\omega^{2}\left[M_{h h}\right]+(1+i g)\left[K_{h h}\right]-\frac{1}{2} \rho V^{2}\left[Q_{h h}(M, k)\right]\right]\left\{u_{h}\right\}=\{P(\omega)\}(3)
$$

The MSC/NASTRAN full span model was used for further discrete gust analysis. The DLM was used to compute the unsteady aerodynamics. The parameters for a single flight condition are listed in Table. The gust velocity used in the analysis is taken from reference [8].

Table. Flight conditions

\begin{tabular}{|l|c|}
\hline Altitude & $1000.0 \mathrm{~m}$ \\
\hline True air speed & $44.0 \mathrm{~m} . \mathrm{s}^{-1}$ \\
\hline Dynamic pressure & $1076.06 \mathrm{~Pa}$ \\
\hline Gust velocity & $15.0 \mathrm{~m} . \mathrm{s}^{-1}$ \\
\hline
\end{tabular}

The following simplified diagram shows the algorithm used in the computational process [3]. The computations are performed in the frequency domain. In order to obtain the results in time domain an inverse Fourier Transformation needs to be implemented. 


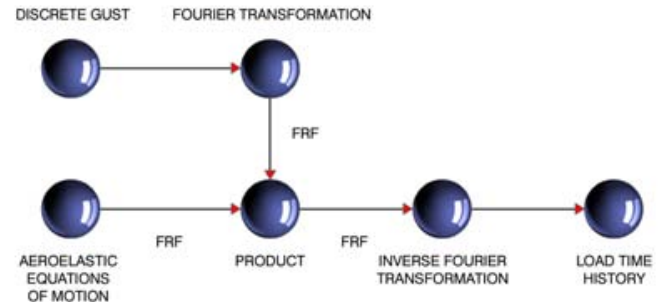

Fig 5. Computational process

\section{Results and discussion}

The estimation of the dynamic response parameters is one of the basic tasks in the line of design processes. The classical analytical method supports procedures derived to forecast flying qualities and performances of rigid aeronautical structures. The purpose of the work was to compare the results obtained from classical analytical solutions and a complex numerical simulation.

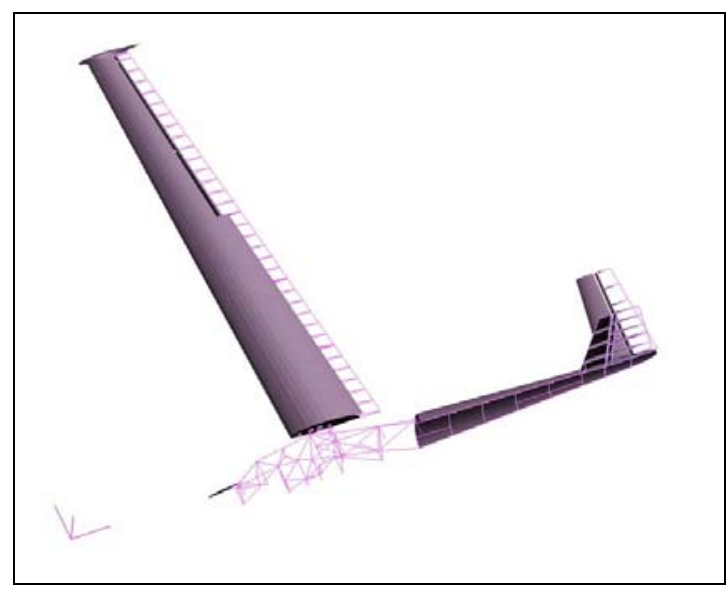

Fig 6. Semi span FEM model

For purposes of this analysis, several points have been selected for outputting acceleration time history. However, further discussion will be directed solely towards results obtained from monitoring points placed at the C.G. and wing tip - close to the main spar. Resulting graphs depicting acceleration time history can be seen in the accompanying picture. Discrete gust analysis using the Pratt-Walker formula presents a simple problem of inserting appropriate values into the equation (1).

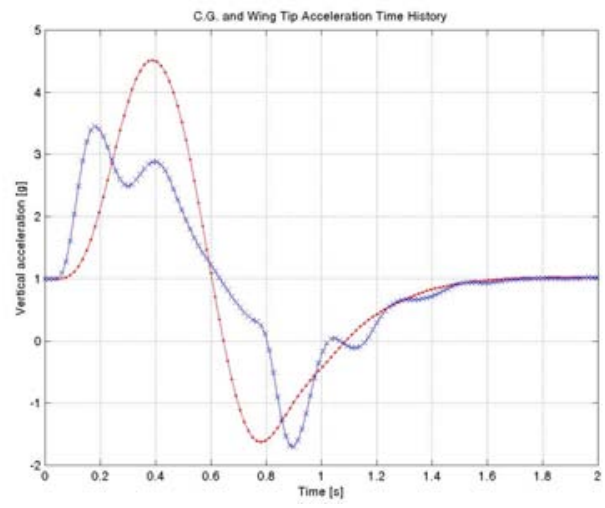

Fig 7. Acceleration history
By comparing the two graphs we can point at the elastic nature of the investigated aeronautical structure. The most severe acceleration occurs at the airplane's center of gravity. The wingtip monitoring station encounters less acceleration, but its time history presents a more complex phenomenon. Inserting the line representing Pratt-Walker's static solution into the graph of C.G. acceleration history provides a visual tool for comparative analysis. Comparing the specific values, a fairly good agreement between both discrete gust analysis methods can be shown. The advantage of the discrete gust numerical simulation lies in the solution of its time history. The nature of the glider's motion (dynamic behavior) is visualized in the time history graph. It is a very important conclusion from the flight mechanics point of view. Since the discrete gust formula gives only quantitative, time-independent (stationary) results, the utilization of advanced dynamic analysis provides the end user with time-related data. Considering this information, conclusions regarding the stability of the system can be formulated.

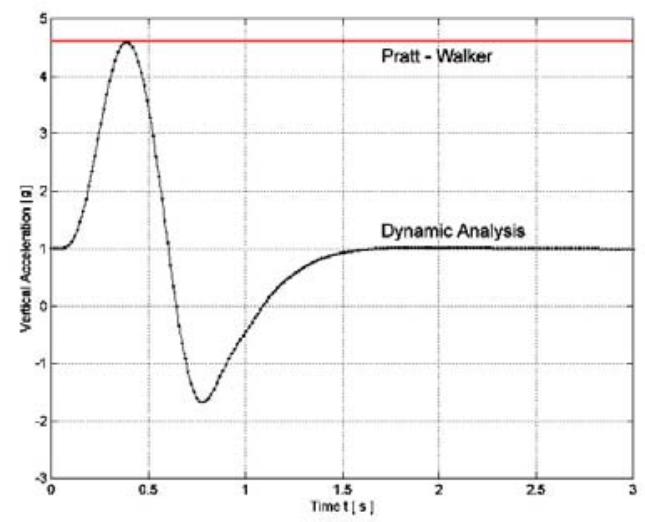

Fig 8. Gust analysis results

\section{Conclusions}

The results clearly indicate the qualitative and quantitative nature of the computational response analysis. The type of modeling performed shows advantages in the 'description' of complex structures. Compared to the analytical solution, which reduces the entire process to a straightforward manipulation with time proven graphs and tables, the numerical simulation offers a more complex description of the dynamic processes investigated. No assumptions neglecting basic geometrical structural properties have been done. A complex simulation, in contrast to the analytical solution offering only one estimated parameter, allows monitoring selected quantities in time domain, thus offering a tool for a visual qualification of the investigated process. The assumption of a sufficient 'rigidity' of the investigated system was confirmed by comparing the results of both analyses. The analytical solution and the 'competing' mathematical simulation are complementary tools in the case of classical light aeronautical structure. Complex structural models could be further used for optimization 
purposes as well as for other static and dynamic tasks. The complexity of modeling paid back in the form of the highly illustrative scope of results.

\section{References}

1. Bisplinghoff R.L., Ashley H., Halfman R.L. Aeroelasticity. - New York: Dover Publications, 1996.

2. Donely P. Summary of Information Relating to Gust Loads on Airplanes // NACA Rep. -1950. - No 997.

3. Eight NASTRAN User's Colloquium, NASA-CP2131.

4. Hoblit F.M. Gust Loads on Aircraft: Concepts and Applications / AIAA Education Series. - Washington: AIAA, 1988.
5. Hodges D.H., Pierce G.A. Introduction to Structural Dynamics and Aeroelasticity. Cambridge Aerospace Series. -2002.

6. JAR-22: Sailplanes and Powered Sailplanes. - 2001.

7. Locke J.E. Balanced Continuous Turbulence Gust Loads Using Solution 146. - 1999.

8. Naser A.S., Pototzky A.S., Spain C.V. Response of Alliance 1 Proof-of-Concept Airplane Under Gust Loads. NASA/CR-2001-210649. - 2001.

9. Pratt K.G., Walker W.G. A Revised Gust-Load Formula and a Re-Evaluation of V-G Data Taken on Civil Transport Airplanes From 1933 to 1950. // NACA Report. - 1954. - No 1206.

10. Rodden W.P., Johnson E.H. MSC/NASTRAN Aeroelastic Analysis User's Guide / The MacNealSchwendler Corporation. 